\title{
Efficacy of extracranial stereotactic body radiation therapy (SBRT) added to standard treatment in patients with solid tumors (breast, prostate and non-small cell lung cancer) with up to 3 bone-only metastases: study protocol for a randomised phase III trial (STEREO-OS)
}

Sébastien Thureau ${ }^{1,2^{*}}$, Vincent Marchesi ${ }^{3}$, Marie-Hélène Vieillard ${ }^{4}$, Lionel Perrier ${ }^{5}$, Albert Lisbona ${ }^{6}$, Marianne Leheurteur ${ }^{7}$, Jean Tredaniel ${ }^{8}$, Stéphane Culine ${ }^{9,10}$, Bernard Dubray $^{1,2}$, Naïma Bonnet ${ }^{11}$, Bernard Asselain ${ }^{11}$, Julia Salleron $^{12}$ and Jean-Christophe Faivre ${ }^{3}$ (I)

\begin{abstract}
Background: Stereotactic Body Radiation Therapy (SBRT) is an innovative modality based on high precision planning and delivery. Cancer with bone metastases and oligometastases are associated with an intermediate or good prognosis. We assume that prolonged survival rates would be achieved if both the primary tumor and metastases are controlled by local treatment. Our purpose is to demonstrate, via a multicenter randomized phase III trial, that local treatment of metastatic sites with curative intent with SBRT associated of systemic standard of care treatment would improve the progression-free survival in patients with solid tumor (breast, prostate and non-small cell lung cancer) with up to 3 bone-only metastases compared to patients who received systemic standard of care treatment alone.
\end{abstract}

Methods: This is an open-labeled randomized superiority multicenter phase III trial. Patients with up to 3 bone-only metastases will be randomized in a 1:1 ratio.between Arm A (Experimental group): Standard care of treatment \& SBRT to all bone metastases, and Arm B (Control group): standard care of treatment.

For patients receiving SBRT, radiotherapy dose and fractionation depends on the site of the bone metastasis and the proximity to critical normal structures. This study aims to accrue a total of 196 patients within 4 years.

(Continued on next page)

\footnotetext{
* Correspondence: sebastien.thureau@chb.unicancer.fr

${ }^{1}$ Radiation Oncology \& Medical Physics Department, Henri-Becquerel Comprehensive Cancer Center, rue d'Amiens, F-76 000 Rouen, France

${ }^{2}$ EA4108 Quantlf Litis, University of Rouen, 22 boulevard Gambetta, 76000 Rouen, France

Full list of author information is available at the end of the article
}

(c) The Author(s). 2021 Open Access This article is licensed under a Creative Commons Attribution 4.0 International License, which permits use, sharing, adaptation, distribution and reproduction in any medium or format, as long as you give appropriate credit to the original author(s) and the source, provide a link to the Creative Commons licence, and indicate if changes were made. The images or other third party material in this article are included in the article's Creative Commons licence, unless indicated otherwise in a credit line to the material. If material is not included in the article's Creative Commons licence and your intended use is not permitted by statutory regulation or exceeds the permitted use, you will need to obtain permission directly from the copyright holder. To view a copy of this licence, visit http://creativecommons.org/licenses/by/4.0/. The Creative Commons Public Domain Dedication waiver (http://creativecommons.org/publicdomain/zero/1.0/) applies to the data made available in this article, unless otherwise stated in a credit line to the data. 
(Continued from previous page)

The primary endpoint is progression-free survival at 1 year, and secondary endpoints include Bone progression-free survival; Local control; Cancer-specific survival; Overall survival; Toxicity; Quality of life; Pain score analysis, Cost-utility analysis; Cost-effectiveness analysis and Budget impact analysis.

Discussion: The expected benefit for the patient in the experimental arm is a longer expectancy of life without skeletal recurrence and the discomfort, pain and drastic reduction of mobility and handicap that the lack of local control of bone metastases eventually inflicts.

Trials registration: ClinicalTrials.gov NCT03143322 Registered on May 8th 2017. Ongoing study

Keywords: MeSH: Oligometastases, Bone metastases, Stereotactic radiotherapy, Neoplasm metastasis, Radiosurgery, Lung neoplasms, Prostatic neoplasms, Breast neoplasms

\section{Background}

Cancer with bone metastases compared to other metastatic sites is considered as associated with a better prognosis, particularly for breast and prostate cancer despite a significant impact on the patient's quality of life and autonomy. Many randomized controlled trials have demonstrated the efficacy of first-line palliative conventional radiotherapy for painful bone metastases [1-4]. The advent of novel imaging techniques allows an increasing an early detection and diagnosis of oligometastatic disease. In clinical practice the possibility to identify a few number of lesions, better defined recently by ASTRO, EORTC and ESTRO, it is considered an intermediate phase of tumor spread with limited metastatic capacity $[5,6]$.

Patients with oligometastatic disease have improved survival compared with those with high- volume metastatic disease. While systemic treatment is the standard of care. Stereotactic radiotherapy is a highly accurate technique initially developed for performing the radiosurgery of lesion in patients for whom it was deemed be to difficult to proceed to classical excision surgery. In the context of patients with oligo-metastatic cancer, high doses of radiation in stereotactic conditions can be safely delivered in bone metastases with increased probability of local control. Local control of bone metastasis is similar to than other localizations with a good control of pain $[7,8]$ and patients with oligometastasis had a longer survival with better local control for spine metastasis [9] and delay progression, and thereby postpone the need for further systemic treatment $[10,11]$.

In combination with curative-intent treatment to the primary tumor and systemic therapy, we hypothesize that an improved local control in the bone genuine de novo synchronous or metachronous oligometastatic disease would be associated with an increased ProgressionFree Survival (PFS) and Overall Survival (OS).

Two ancillaries studies are associated:

- Biological ancillary study which search for pre-SBRT and follow-up markers (serum CTX and bone ALP) associated with skeletal-related events and bone progression disease.
- Economic ancillary study.

\section{Methods/design}

This is an open-labeled randomized superiority multicentric phase III studyin French academic hospital. Patients will randomized in a 1:1 ratio between (Fig. 1):

Arm A (experimental group): Standard care of treatment (systemic treatment suitable for primary cancer) and SBRT to the bone metastases.

Arm B (control group): Standard care of treatment (systemic treatment suitable for primary cancer) alone.

The sample size allows 2 stratification factors at randomization: date of metastases (synchronous vs metachronous) and primary tumor site (prostate vs breast vs lung). All patients will be randomized after completion of the primary tumor treatment for synchronous or metachronous metastasis. The randomization will be minimized by the following stratification factors. Minimization aims to ensure treatment arms are balanced with respect to predefined patient factors as well as for the number of patients in each group.

\section{Objectives}

To assess the impact of SBRT on Progression Free Survival in patients with solid tumor with up to bone-only metastases, compare to patients who received standard of care treatment alone.

\section{Primary endpoint}

The primary endpoint is the 1-year PFS. Progression Free Survival PFS is defined as the time from randomization until the date of the first objective progression or death (whatever its cause in the absence of progression). Stereo-OS protocol will allow a specific focus on skeletal metastases and is thus expected to bring a clear-cut assessment of the added benefit of stereotactic radiotherapy on PFS. 


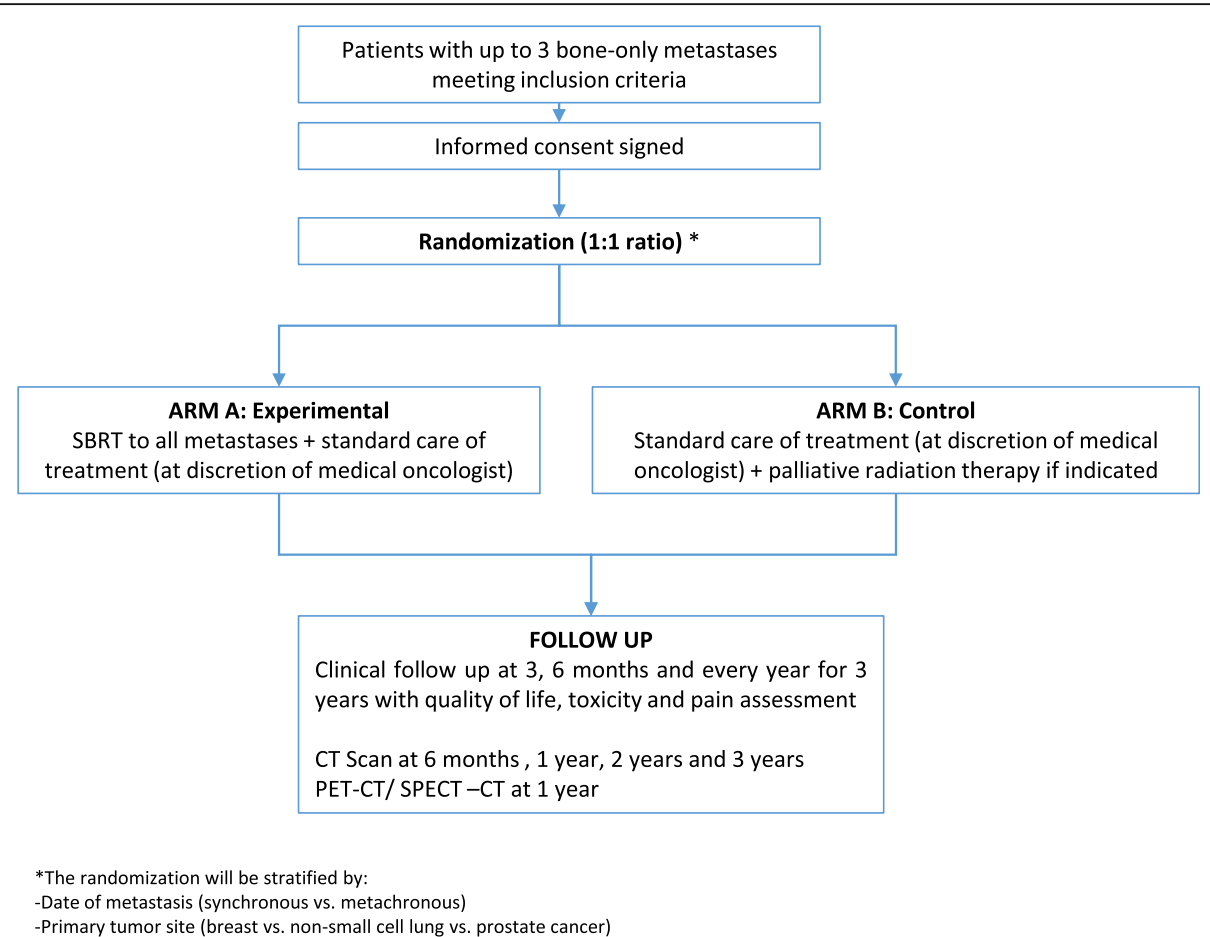

Fig. 1 Study Design. Footnotes: SBRT: Stereotactic Body radiation Therapy; CT Scan: Computed tomography Scann; PET: Positron Emission Tomography; SPECT-CT: Single Photon Emission Tomography-Computed Tomography

\section{Secondary endpoints}

The secondary endpoints are as follow:

- PFS at 2 and 3 years defined as the time from randomization until the date of the first objective progression or death

- Bone progression free-survival (BPFS) at 1 year defined as the time from the date of randomization to the date of documented bone progression at the level at the entire skeleton.

- Local control (LC) at 1 year defined as the time from the date of randomization to the date of a documented local event at the level of bone oligometastases presents at randomization.

- Cancer-specific survival (CSS) at 1,2 and 3 years defined as the time from the date of randomization to the date of documented death from cancer or complication from the treatment.

- Overall survival (OS) at 1, 2 and 3 years defined as the time from the date of randomization to the date of documented death from any cause.

- Acute and late Toxicity at 1,2 and 3 years assessed by the National Cancer Institute Common Toxicity Criteria (NCI CTC) version 4.0.

- Quality of Life assessed using self-administered questionnaires (EORTC-QLQ-C30, EORTC-QLQBM22, and EQ-5D-3L) [12-14].

- Pain score evaluated accordingly to Numeric Scale.
- The Cost- Utility /Cost-Effectiveness / Budget Impact analysis performed on QALYs (QualityAdjusted Life Years) and ICERs (Incremental CostEffectiveness Ratios) calculation based on EQ-5D-3L questionnaire.

\section{Eligibility criteria}

The inclusion criteria are as follow:

- Patients older than 18 years

- Good general condition: WHO performance status $\leq 1$

- Patients with histological proof of breast, non-small cell lung, or prostate cancer

- Absence of co-morbidity contra-indicating radiochemotherapy or surgery

- Primary tumor accessible to curative-intent treatment (surgery, chemoradiation...) for patients with synchronous metastases (ie randomization after treatment of primitive cancer)

- Patients with between 1 and 3 synchronous or metachronous bone metastases as defined by NaFPET or conventional SPECT-CT scan and spinal MRI (if necessary) within 6 weeks before randomization

- Bones metastases treatable by SBRT

- Primary cancer considered to be controlled or accessible to curative-intent treatment (surgery, 
chemoradiation...) in case of locoregional reccurence for metachronous bone oligo-metastatic disease

- Women of childbearing potential and male patients must agree to use adequate contraception for the duration of study participation and up to 3 months following completion of therapy;

- Patients who have received the information sheet, dated and signed the informed consent form

- Affiliated to the social security system

The non-inclusion criteria are as follow:

- Visceral metastases as defined by FDG-PET (or FCholine-PET for prostate cancer) and cerebral CT or MRI performed

- Previous systemic therapy for metastasis for patients with metachronous metastasis. Prostate and breast cancer patients remain eligible if hormonal treatment was initiated 6 months before enrollment.

- All bone metastasis requiring surgical treatment (spinal cord compression, fracture, ...)

- More than 3 bone metastases as defined by NaFPET or conventional SPECT-CT scan and spinal MRI (if spinal bone metastases on NaF-PET)

- Previous cancer within the 5 years before inclusion (except basal cell carcinoma of the skin, in situ carcinoma of the uterine cervix)

- Previous radiotherapy on bone metastasis (e.g: antalgic radiotherapy)

- Patient enrolled in another therapeutic trial

- Pregnant women or breast feeding mothers,

- Hypersensitivity to the active substance (FDG and $\mathrm{NaF}$ or F-Choline for prostate cancer) or to any of the excipients

- Contraindication to MRI (in case of spinal metastases)

- Patients deprived of liberty or placed under the authority of a tutor. Patients with any psychological, familial, sociological or geographical condition potentially hampering compliance with the study protocol and follow-up schedule; those conditions should be discussed with the patient before registration in the trial. Patients unable to understand the purpose of the study (language, etc.).

\section{Evaluation and randomization}

Prior to randomization, a complete history and physical examination is required. Histologically confirmation of malignancy is required, with metastatic disease detected on imaging.

The mechanism of implementing the allocation sequence (stratified randomization) is generated by computer. Clinical research assistant generate the allocation sequence and physician enroll and assign participant to intervention.

Patients must be restaged within 6 weeks before randomization, including brain CT or MRI and CTTAP. For all patients with a suspicion of spinal bone metastases, a spinal MRI is mandatory.

The patients will also benefit from PET-CT exams before treatment. PET1 F-Choline, NaF and FDG are standard examinations for metastases staging; and PET2 FDG or F-Choline for evaluation. If NaF-PET cannot be done, patients will have a "conventional" biphosphonates SPECT-CT with a tomographic exploration from the vertex to mid-thigh in double or triple field instead.

Patients with prostate cancer are also required to have PSA blood test. A negative pregnancy is required for women of child-bearing age (Table 1).

The present research project will be developed for 48 months, during which specific milestones will be achieved and documented (Table 1).

\section{Interventions/treatments}

Arm A

In case of SBRT, the acceptable regimens are $35 \mathrm{~Gy} / 7$ fractions / 3 fractions per week or $27 \mathrm{~Gy} / 3$ fractions / 3 fractions per week. An interval of at least $24 \mathrm{~h}$ should be kept between two consecutive fractions. Treatment planning and delivery will be performed using dedicated system. Strict quality assurance protocols will be implemented to ensure the accuracy in dose delivery.

All patients in Arm A will undergo planning CT simulation. CT simulation must be performed in the treatment position with adequate immobilization devices and the fiducials must be placed for stereotactic targeting if necessary. The use of intravenous contrast is recommended. Axial reconstructions will be required with slice thickness of around $1 \mathrm{~mm}$. MRI, and PET if needed will be merged with CT simulation at the target volume on the simulation CT.

The Gross Tumor Volume (GTV) corresponds to the tumor volume visible on the simulation CT taking into account the information provided by the clinical examination and the other imaging modalities. (MRI is mandatory for spinal metastasis and experimental arm).

The Clinical Target Volume (CTV) corresponds to the macroscopic spread. Its limits are defined by Cox et al. for spine metastases or by CTV $=\mathrm{GTV}+5 \mathrm{~mm}$ for the other bones [15].

The PTV is obtained by adding a margin around the CTV to take into account the set-up uncertainty during radiotherapy delivery with $\mathrm{PTV}=\mathrm{CTV}+2 \mathrm{~mm}$. This margin can be reduced to $0 \mathrm{~mm}$ close to the spinal cord. Depending on the SBRT equipment, the beam aperture can be set to $2-3 \mathrm{~mm}$ beyond the CTV to provide 
Table 1 Timeline and follow up of the study

\begin{tabular}{|c|c|c|c|c|c|c|c|c|}
\hline & Screening & Randomization & Treatment & Follc & & & & \\
\hline & & & & M3 & M6 & $\mathrm{Y} 1$ & Y2 & Y3 \\
\hline History \& physical & $x$ & & $x$ & $x$ & $x$ & $x$ & $x$ & $x$ \\
\hline Brain $\mathrm{CT}$ or MRI & $x$ & & & & $x$ & $x$ & $x$ & $x$ \\
\hline TAP CT & $x$ & & & & $x$ & $x$ & $x$ & $x$ \\
\hline FDG PET or F-Choline PET & $x$ & & & & & $x$ & & \\
\hline PET or SPECT-CT & $x$ & & & & & $x$ & & \\
\hline Spinal MRI (if spinal bone metastases) & $x$ & & & & $x$ & $x$ & & \\
\hline Mammography (breast cancer) & $x$ & & & & $x$ & $x$ & $x$ & $x$ \\
\hline Histological confirmation & $x$ & & & & & & & \\
\hline Quality of Life & $x$ & & $x$ & $x$ & $x$ & $x$ & $x$ & $x$ \\
\hline Toxicity Assessment & & & $x$ & $x$ & $x$ & $x$ & $x$ & $x$ \\
\hline Pain Assessment & $x$ & & $x$ & $x$ & $x$ & $x$ & $x$ & $x$ \\
\hline Economic Study & & & $x$ & $x$ & $x$ & $x$ & $x$ & $x$ \\
\hline sCTX/ bALP Evaluation & $x$ & & $X$ & $x$ & $x$ & $x$ & & \\
\hline
\end{tabular}

MRI Magnetic Resonance Imaging; TAP-CT Computed tomography thorax-abdomen-pelvis; FDG-PET Fluorodesoxyglucose-Positron Emission Tomography; FCholine-PET Fluor-Choline-Positron Emission Tomography; PET Positron Emission Tomography; SPECT-CT Single Photon Emission Tomography-Computed Tomography; sCTX serum C-Telopeptide cross-link of type 1 collagen; bALP bone ALkaline Phosphatase

adequate dose coverage. This margin can be reduced to $0-1 \mathrm{~mm}$ close to the spinal cord.

Prescription must be defined on $80 \%$ isodose or higher. At least $90 \%$ of the PTV should receive the prescribed dose. A coverage of $<90 \%$ of the PTV will be considered as Acceptable Deviation, and coverage of $<$ $80 \%$ of the target volume as an unacceptable deviation. At least $90 \%$ of the GTV should receive the prescribed dose. A coverage of $<90 \%$ of the GTV will be considered as Acceptable Deviation, and coverage of $<80 \%$ of the target volume as an unacceptable deviation.

The organs at risk (OAR) of complication include all normal tissues located in the vicinity of the target or in the beams path. Specific dose-volumes constraints, defined by Timmermann, have to be respected to avoid unacceptable toxicity [16]. In the Experimental group with SBRT, all relevant OARs must be delineated. As for the spinal cord, two volumes have to be delineated: A "conventional" volume is delineated on the simulation CT after registration of MRI (T2-weighted and T1weighted with contrast). The contour should be drawn at least $10 \mathrm{~cm}$ above and below the CTV, as recommended by NRG Oncology protocols. A "partial" volume is specific to the present study (Table 2). The contour drawn as above ("conventional" volume) extends 5-6 $\mathrm{mm}$ above and below the CTV [17].

For the others OAR not listed in Table 2, investigators must refer to AAPM Task Group 101 report. It is important to deliver as low a dose as possible in the organs at risk while assuring that a sufficient dose to the target volume is delivered.

\section{Arm B}

In the Control group (without SBRT), palliative radiotherapy on bone metastases is allowed if necessary (pain, fracture, spinal cord compression ...).

The acceptable regimens for patients in Arm1 are as follows as per consensus guidelines [18]:

- 30 Gy / 10 fractions / 5 fractions per week

- 24 Gy / 6 fractions / 5 fractions per week

- 20 Gy / 5 fractions / 5 fractions per week

Table 2 OAR Constraints

\begin{tabular}{llll}
\hline & 3D RT & SBRT 3 fractions & SBRT 5 fractions \\
\hline Spinal cord & Dmax $<45 G y$ & $V 18 \leq 10 \%$ for « partial » volume & V23 $\leq 10 \%$ \\
& (standard fractionation) & $V 18 \leq 0.35 \mathrm{~cm}^{3}$ for « conventional » volume & $V 23 \leq 0.35 \mathrm{~cm}^{3}$ \\
Cauda equina & $\mathrm{V} 12 \leq 1.2 \mathrm{~cm}^{3}$ for « conventional » volume & $V 14.5 \leq 1.2 \mathrm{~cm}^{3}$ \\
& & $V 22.5<5 \mathrm{~cm}^{3}$ & $V 30<5 \mathrm{~cm}^{3}$ \\
Sacral plexus & $\mathrm{D} 2 \%<24 \mathrm{~Gy}$ & $\mathrm{D} 2 \%<32 \mathrm{~Gy}$ \\
\hline
\end{tabular}


- 8 Gy / 1 fraction

The organs at risk are not delineated in the control group except for the spinal cord (Table 2).

\section{Ethical consideration and study registration}

This Study has been approved by Nord-Ouest I Ethics Committee and French Regulatory authorities (ANSM). French ethics committee (CPP Nord-Ouest I) has approved this protocol on October 13th 2016 (reference number: CPP 02/016/2016). The study will be performed in accordance with the Declaration of Helsinki and will comply to the International Conference on Harmonization and Good Clinical Practice and General Data Protection Regulation (GDPR). This study funded by grants from French Cancer Institute (Institut National du Cancer, 52, avenue André Morizet, F-92513 Boulogne Billancourt Cedex) though PHRC program (grant number: PHRC-K 2015-149) that has no role in the collection, analysis, interpretation of results or writing of manuscripts. The study has been registered at clinicaltrials.gov (ClinicalTrials.gov NCT03143322 Registered on May 8th 2017).

Patient information and informed consent from the patient must be handled in accordance with the French regulation. Prior to the participation of a patient in the trial, this patient will be informed both verbally and in writing about the objectives of the trial, its methods, anticipated benefits and potential risks and the discomfort to which they may be exposed. The informed consent form for study and ancillaries studies, must be personally dated and signed by the patient and investigator.

\section{Quality Assurance of Radiation Therapy}

In order to ensure patient safety and effective treatment delivery, a quality assurance protocol is implemented. Prior to opening the study, each participant centre will have to validate a dummy run by delineate targets and OARs and produce a treatment plan from a benchmark case on Aquilab software. The Quality Assurance committee will evaluate the dummy run. Patient inclusions by a centre will be allowed only after full validation. Afterwards, each participating centre will have to send planning and contouring information for the first included patient for review before the start of the radiotherapy. The QA committee according to the protocol recommendations in terms of delineation, dose constraints and treatment workflow will evaluate this treatment plan. At the end of treatment, each participating centre will have to send final review of the RT data for all included patients in SBRT arm for review. The QA committee, according to the protocol recommendations in terms of delineation, dose constraints and treatment workflow will evaluate the treatment plan for Quality Assurance.

\section{Systemic treatment}

The patients will receive systemic standard of care for the metastases according to current oncological national recommendations and international guidelines.

\section{Follow-up}

Patients will be seen at 3 months, 6 months post randomization and then every year for 3 years.

(Table 1). At each visit, the oncologist will conduct a history and physical examination, and assess CTC-AE toxicities, pain and quality of life. CT head, chest, abdomen and pelvis will be repeated at 6 months, then every year for 3 years. PET-CT and SPECT-CT will be repeated at 1 year. Additional Imaging should be carried out at the discretion of the physician. Additional treatment (e.g. further chemotherapy) is at the discretion of the oncologists.

\section{Measurement of response}

Progression-free survival will be measured as time to either progression or death, whichever occurs first. Efficacy Evaluation will be performed at baseline, at 6 months, at 1 year, then subsequently every year until the end of study, using the same imaging method as for baseline (CT scan or MRI or PET if indicated).

Lesion response will be evaluated in this study using the RECIST criteria, PERCIST Criteria and MD Anderson Criteria [19].

\section{Statistical analysis}

Sample size calculation: 196 patients will be randomized between 2 arms (in a 1:1 ratio). The primary endpoint is the 1-year Progression Free Survival (PFS). In the standard arm, the expected progression-free survival at 1 year is $30 \%$ ( $10 \%$ for lung cancer, $50 \%$ for breast cancer and $30 \%$ for prostatic cancers). In the experimental arm, we anticipate a $20 \%$ absolute increase (i.e. 30,70 and 50\%, respectively). According to Freedman's method, accepting a two-tailed type I error of $5 \%$ and an $80 \%$ power (type II error of 20\%), 178 patients ( 2 X 89) need to be recruited and 106 events need to be observed for the primary analysis. Assuming a 10\% withdrawal rate, a total of 196 patients will need to be included.

Data analysis: All patients will be randomized after completion of the primary tumor treatment for synchronous or metachronous metastasis. The randomization will be stratified by date of metastasis (synchronous vs. metachronous) and primary tumor site (breast vs. non-small cell lung vs. prostate cancer). Statistical analysis will be performed on all the randomized patients, accordingly to the intent to treat (ITT) arm, whatever the actually received treatment. Patients without progression and alive at the time of analysis will be censored at the time of the latest assessment. 
Primary Endpoint Analysis: Progression Free Survival PFS is defined as the time from randomization until the date of the first objective progression accordingly to RECI ST or death (whatever its cause in the absence of progression). Patients without progression and alive at the time of analysis will be censored at the time of the latest assessment. The primary analysis will take place when 106 events have been observed. This analysis will use the logrank test, stratified on the primary location and the time of onset of the bone metastases (synchronous vs metachronous). Cox regression model will also be used to perform a sensitivity analysis taking into account the usual prognostic factors. The hazard ratio will be given with its $95 \%$ confidence interval. A $p$ value $<=0.05$ will be considered as statistically significant. A secondary analysis will also be performed, using the Fine and Gray model in order to take into account the competing risks (relapse in the bone, relapse in another site, death), and so to analyse the impact of the different PFS events on the trial results.

\section{Data safety monitoring committee}

An Data Safety Monitoring Committee (DSMC), with expertise and experience in the pathology, and without direct involvement in the conduct of the trial, will be set up specifically to guarantee effective protection of patients, insure the ethical conduct of the trial, benefit/risk ratio of the trial, and to ensure the independent review of the scientific results during the trial and at the end of the trial. The DSMC will meet after 60 patients are accrued in the experimental arm to review toxicity outcomes and compliance.

The DSMC may recommend the early termination of the trial if one of the following conditions is met: an unacceptable toxicity or all available data from the trial or any other source of information are sufficiently convincing to influence the therapeutical practices of the majority of clinicians. The DSMC has only a consultative role; it will inform the sponsor who will decide whether the DSMC recommendation will be followed. An adverse event (AE) is defined as any untoward medical occurrence, in a patient or clinical trial subject treated by a medicinal product and which does not necessarily have a causal relationship with this treatment. The assessment of whether there is a reasonable causal relationship is made by the investigator. Notification must be carried out immediately by fax to the R\&D UNICANCER pharmacovigilance unit by sending the form "notification of a Serious Adverse Effects", located in the Investigator Master File, completed as precisely as possible, dated and signed by the physician-investigator.

\section{Biological ancillary study}

The follow-up of bone metastasis evaluation is difficult by means of anatomic imaging (CT-scan). There is a need to identify biologic markers that would predict bone disease progression (DP) and risk of skeletalrelated events (SREs) as sequelae of bone metastases. As this ancillary study, we have chosen to explore s-CTX and bone alkaline phosphatase (b-ALP) to better quantify response to SBRT vs standard treatment [20-22]. Predictive value of these biomarkers at different points will be assessed on disease control rate (DCR) [23-26]. To perform this study, blood samples (sCTX and bALP: $7 \mathrm{ml}$ in a dry tube will be collected before the beginning of treatment, at the end of treatment, at 3 months, 6 months and 1 year after randomization.

\section{Economic ancillary study}

Cost-utility, cost-effectiveness, and budget impact analyses performed on QALYs (Quality-Adjusted Life Years) and ICERs (Incremental Cost-Effectiveness Ratios) calculation based on EQ-5D-3L questionnaire will provide useful and complementary information in order (i) to recommend the best strategy to adopt; (ii) to estimate the budget impact on the French National Health Insurance of the generalization of the cost-effective strategy [27-29]. Analyses will be performed comparing SBRT versus conventional treatment without SBRT in solid tumors patients with $\leq 3$ bone-only metastasis. Incremental costs between both arms will be compared to incremental health improvement.

\section{Discussion}

Stereotactic radiotherapy is a routine treatment for brain, hepatic and lung metastases with increased probability of local control. However, Loco Regional Control is similar to bone metastasis than other localizations with $95 \%$ and a good control of pain and patients with oligometastasis had a longer survival with better local control for spine metastasis $[10,11]$.

The tools required for high precision radiotherapy are now commercially available and implemented in many radiotherapy departments. Sufficient clinical experience has been acquired so that large randomized trials can confidently be launched. Current knowledge is mainly based on retrospective and prospectives non-randomized studies, thus suffering from heterogeneous population and inappropriate sample power. The oligometastatic concept has been developed in the last years and recent trials show interesting results on adding Metastase Directed Treatment (MDT) (such as radiotherapy or surgery) at standard therapy. However, despite encouraging data, some authors remain skeptical that MDT will delay the use of systemic treament.

In a randomized phase II trial, named SABR-COMET, a benefit in OS in the SBRT arm for the oligometastatic setting compared to palliative treatment was demonstrated (41 months vs 28 months) [30]. In oligometastatic 
NSCLC, Gomez et al., that conducted a randomized phase II trial on oligometastatic NSCLC, reporting a significant PFS benefit with LCT (both radiotherapy or surgery) vs maintenance systemic therapy (14.4 months vs 4.4 months respectively). In oligometastic prostate cancer, HORRAD, STAMPEDE, STOMP and ORIOLE studies have shown encouraging results [31-34]. In oligometastatic breast cancer, Steven David et al. demonstrated that SABR is feasible, well tolerated and effective in selected patients with bone-only oligometastatic disease [35].

Two other randomized trials, SARON and CORE investigate the same approach in different clinical settings with oligometastatic patients [36]s. With respect to these two studies, Stereo-OS will allow a specific focus on skeletal metastases only and is thus expected to bring a clear-cut assessment of the added benefit of stereotactic radiotherapy on PFS in the especially dedicated treatment of a highly frequently occurring type of metastases in solid tumor cancers.

In a recent review of Faiez Al-Shafa et al., on Ongoing Trials of Stereotactic Ablative Radiotherapy for Oligometastatic Cancers, A minority of trials were randomized in design $(n=17,27 \%)$. While most studies allowed for metastases from multiple primary disease sites $(n=$ $22,34 \%)$, the most common was prostate $(n=13,15 \%)$, followed by breast, gastrointestinal, non-small cell lung cancer (NSCLC), and renal ( $n=6,9 \%$ each). In studies with a solitary target site, the most common was liver $(n=6,9 \%)$ followed by lung $(n=3,5 \%)$. The most common primary endpoints were progression-free survival (PFS) $(n=20,31 \%)$ and toxicity $(n=10,16 \%)$. A combined strategy of systemic therapy and SABR was an emerging theme $(n=23,36 \%)$, with more recent studies specifically evaluating SABR and immunotherapy $(n=9$, 14\%) [37].

The safety and efficacy of SABR as oligometastasisdirected treatment is increasingly being evaluated within prospective clinical trials. These data are awaited to compliment the abundance of existing observational studies and to guide clinical decisionmaking.

\footnotetext{
Abbreviations

SABR: Stereotactic ablative radiotherapy; WBRT: Whole brain radiotherapy; CTC-AE: Common Terminology Criteria for Adverse Events; CT: Computed tomography; PET: Positron emission tomography; MRI: Magnetic resonance imaging; GTV: Gross tumor volume; CTV: Clinical target volume; PTV: Planning target volume; PRV: Planning organ at risk volume; PI: Principal investigator; QA: Quality assurance; RECIST: Response evaluation criteria in solid tumors; PERCIST: Positron Emission Tomography (PET) Response Criteria in Solid Tumors; PFS: Progression Free Survival; OS: Overall survival; DSMC: Data Safety Monitoring Committee; ITT: Intent to treat; QALYS: Quality-Adjusted Life Years; ICERs: Incremental Cost-Effectiveness Ratios; QLQ: Quality of Life Questionnaire; Gy: Gray; FDG: Fluorodeoxyglucose; NA-F: Sodium Fluoride; EORTC: European Organisation for Research and Treatment of Cancer; ESMO: European Society for Medical Oncology; INCa: Insitut du Cancer
}

\section{Acknowledgements}

We thanks SFRO (French Society of Radiation Oncology), SFMN (French Society of Nuclear medicine), UNITRAD (Unicancer group of Translational research and development in RADiation oncology) and GEMO (European Society of Bone Metastases) for their support and all centers which are participating in this ongoing study.

Céline BOURGIER (Institut Régional du Cancer Montpellier Val d' Aurelle); Louis GRAS (Centre Leonard De Vinci-DECHY); Stéphane SUPIOT (Institut de Cancerologie de I'Ouest: Rene Gauducheau - SAINT HERBLAIN et Paul Papin ANGERS); Alain TOLEDANO (Clinique Hartmann - LEVALLOIS PERRE); Renaud DE CREVOISIER (Centre Eugene Marquis - RENNES); Béatrice LAFFORGUE (Centre de Cancérologie du Grand Montpellier - Clinique Clémentville); David PASQUIER (Centre Oscar Lambret - LILLE); Yazid BELKACEMI (Hopital Henri Mondor - CRETEIL); Etienne MARTIN (Centre Georges François Leclerc DIJON); Nicolas MAGNE (Institut de Cancérologie Lucien Neuwirth - SAINT PRIEST EN JAREZ); Marie-Pierre SUNYACH (Centre Léon Bérard - LYON); Benjamin SCHIPMAN (Institut de Cancérologie de Bourgogne - DIJON); PierreEtienne CAILLEUX (Pôle Sante Leonard De Vinci - CHAMBRAY LES TOURS); Vincent VINH-HUNG (CHU de Martinique - Hôpital Clarac-FORT DE FRANCE); Stéphanie SERVAGI-VERNAT (Institut Jean Godinot - REIMS); Laurence GONZAGUE CASABIANCA (Institut Paoli Calmettes - MARSEILLE); Claire IRIART (Centre Hospitalier Universitaire Grenoble Alpes - GRENOBLE); Adeline PETIT (Institut Bergonié - BORDEAUX); Philippe FOURNERET (Hôpital Métropole Savoie-CHAMBERY); Antoine ARNAUD (Institut Sainte Catherine - AVIGNON); Igor LATORZEFF (Clinique Pasteur - TOULOUSE); Emilie BONNET (Centre Marie Curie - VALENCE); Sabine VIEILLOT (Centre Catalan d' Oncologie - PERPIGNA N); Gilles REGNAULT DE LA MOTHE (Clinique Tivoli Ducos - BORDEAUX); Anne LARROUY (Institut de cancérologie Paris Nord - SARCELLES); Simon DANDOY (Centre Joliot Curie - SAINT MARTIN LES BOULOGNES); Thomas LEROY (Nouvelle clinique des Dentellières - VALENCIENNES).

\section{Authors' contributions}

All of the authors have read and approved the final manuscript and protocol. The authors contributed as follows to the research project: Sébastien Thureau, Jean-Christophe Faivre, Naïma Bonnet (project manager), Marie-Hélène Vieillard (biological ancillary study), Lionnel Perrier (economic ancillary study), Vincent Marchesi and Albert Lisbona (medical physics) designed the study and wrote the manuscript. Bernard Asselain, Julia Salleron performed the statistical analysis and wrote the manuscript. Marianne Leheurteur, Jean Tredaniel, Stéphane Culine, Bernard Dubray provided menthorship and reviewed the manuscript. For the main publication, the first author and writer of the publication will be the study coordinators and the statistician will be the third author. The study coordinators may however designate another person to co- write the publication.

\section{Funding}

This study funded by grants from French Cancer Institute (INCa) though PHRC program (grant number: PHRC-K 2015-149) that has no role in the collection, analysis, interpretation of results or writing of manuscripts.

\section{Availability of data and materials}

Data sharing is not applicable to this article as no datasets were yet generated or analyzed during this ongoing study.

\section{Ethics approval and consent to participate}

French ethics committee (CPP Nord-Ouest I) has approved this protocol on October 13th 2016 (reference number: CPP 02/016/2016).

Patient information and informed consent from the patient must be handled in accordance with the French regulation. Prior to the participation of a patient in the trial, this patient will be informed both verbally and in writing about the objectives of the trial, its methods, anticipated benefits and potential risks and the discomfort to which they may be exposed. The informed consent form for study and ancillaries studies, must be personally dated and signed by the patient and investigator.

The manuscript has undergone peer review by the funding body.

\section{Consent for publication}

Not applicable.

\section{Competing interests}

The authors declare that they have no competing interests. 


\section{Author details}

${ }^{1}$ Radiation Oncology \& Medical Physics Department, Henri-Becquerel Comprehensive Cancer Center, rue d'Amiens, F-76 000 Rouen, France. ${ }^{2}$ EA4108 Quantlf Litis, University of Rouen, 22 boulevard Gambetta, 76000 Rouen, France. ${ }^{3}$ Academic Radiation Oncology \& Brachytherapy Department, Lorraine Institute of Cancerology - Alexis-Vautrin Comprehensive Cancer Center, 6 avenue de Bourgogne, 54519 Vandœuvre-lès-Nancy, France. ${ }^{4}$ Rheumatology Department, University Hospital of Lille, 2 avenue Oscar Lambret, 59000 Lille, France. ${ }^{5}$ UMR CNRS 5824, Léon Bérard Comprehensive Cancer Center, 28 rue laennec, 69373 Lyon, France. ${ }^{6}$ Academic Radiation Oncology \& Brachytherapy Department, Institut de Cancérologie de l'Ouest René Gauducheau Comprehensive Cancer Center, Boulevard Professeur Jacques Monod, 44805 Saint-Herblain, France. ${ }^{7}$ Medical Oncology Department, Henri-Becquerel Comprehensive Cancer Center, rue d'Amiens, 76000 Rouen, France. ${ }^{8}$ Pneumology Department, University Hospital of Paris (Groupe hospitalier Paris Saint-Joseph), 185 Rue Raymond Losserand, 75014 Paris, France. ${ }^{9}$ Medical Oncology Department, University Hospital of Paris (Saint-Louis Hospital), 1 avenue Claude Vellefaux, 75010 Paris, France. ${ }^{10}$ Paris Diderot University, 16 rue Huchard, 75018 Paris, France. "'Unicancer, 101, rue de Tolbiac, F-75654 Paris, France. ${ }^{12}$ Biostatistics Department, Institut de Cancérologie de Lorraine - Alexis-Vautrin Comprehensive Cancer Center, 6 avenue de Bourgogne, F-54519 Vandœuvre-lès-Nancy, France.

Received: 2 October 2020 Accepted: 21 January 2021 Published online: 04 February 2021

\section{References}

1. Chow E, Hoskin P, Mitera G, Zeng L, Lutz S, Roos D, et al. Update of the international consensus on palliative radiotherapy endpoints for future clinical trials in bone metastases. Int J Radiat Oncol Biol Phys. 2012 Apr 1; 82(5):1730-7.

2. McQuay HJ, Carroll D, Moore RA. Radiotherapy for painful bone metastases: a systematic review. Clin Oncol R Coll Radiol G B. 1997;9(3):150-4.

3. Wu JS-Y, Wong R, Johnston M, Bezjak A, Whelan T. Meta-analysis of dosefractionation radiotherapy trials for the palliation of painful bone metastases. Int J Radiat Oncol Biol Phys. 2003 Mar 1;55(3):594-605.

4. Sze WM, Shelley M, Held I, Mason M. Palliation of metastatic bone pain: single fraction versus multifraction radiotherapy - a systematic review of the randomised trials. Cochrane Database Syst Rev Online. 2004;2:CD004721.

5. Guckenberger M, Lievens Y, Bouma AB, Collette L, Dekker A. deSouza NM, et al. characterisation and classification of oligometastatic disease: a European Society for Radiotherapy and Oncology and European Organisation for Research and Treatment of Cancer consensus recommendation. Lancet Oncol. 2020;21(1):e18-28.

6. Lievens $Y$, Guckenberger M, Gomez D, Hoyer M, lyengar P, Kindts I, et al. Defining oligometastatic disease from a radiation oncology perspective: an ESTRO-ASTRO consensus document. Radiother Oncol J Eur Soc Ther Radiol Oncol. 2020;148:157-66.

7. Ryu S, Rock J, Rosenblum M, Kim JH. Patterns of failure after single-dose radiosurgery for spinal metastasis. J Neurosurg. 2004;101(Suppl 3):402-5.

8. Ryu S, Jin R, Jin J-Y, Chen Q, Rock J, Anderson J, et al. Pain control by image-guided radiosurgery for solitary spinal metastasis. J Pain Symptom Manag. 2008;35(3):292-8.

9. Ryu S, Jin J-Y, Jin R, Rock J, Ajlouni M, Movsas B, et al. Partial volume tolerance of the spinal cord and complications of single-dose radiosurgery. Cancer. 2007;109(3):628-36.

10. Zeng KL, Tseng C-L, Soliman H, Weiss Y, Sahgal A, Myrehaug S. Stereotactic body radiotherapy (SBRT) for Oligometastatic spine metastases: an overview. Front Oncol. 2019;9:337.

11. Bedard G, McDonald R, Poon I, Erler D, Soliman H, Cheung P, et al. Stereotactic body radiation therapy for non-spine bone metastases--a review of the literature. Ann Palliat Med. 2016 Jan;5(1):58-66.

12. Aaronson NK, Ahmedzai S, Bergman B, Bullinger M, Cull A, Duez NJ, et al. The European Organization for Research and Treatment of Cancer QLQ-C30: a quality-of-life instrument for use in international clinical trials in oncology. J Natl Cancer Inst. 1993 Mar 3;85(5):365-76.

13. Chow E, Hird A, Velikova G, Johnson C, Dewolf L, Bezjak A, et al. The European Organisation for Research and Treatment of Cancer Quality of Life Questionnaire for patients with bone metastases: the EORTC QLQ-BM22. Eur J Cancer Oxf Engl 1990. 2009;45(7):1146-52.
14. Rabin R, de Charro F. EQ-5D: a measure of health status from the EuroQol group. Ann Med. 2001;33(5):337-43.

15. Cox BW, Spratt DE, Lovelock M, Bilsky MH, Lis E, Ryu S, et al. International spine radiosurgery consortium consensus guidelines for target volume definition in spinal stereotactic radiosurgery. Int J Radiat Oncol Biol Phys. 2012:83(5):e597-605.

16. Timmerman RD. An overview of hypofractionation and introduction to this issue of seminars in radiation oncology. Semin Radiat Oncol. 2008;18(4):215-22.

17. Ryu S, Yoon H, Stessin A, Gutman F, Rosiello A, Davis R. Contemporary treatment with radiosurgery for spine metastasis and spinal cord compression in 2015. Radiat Oncol J. 2015;33(1):1-11.

18. Lutz S, Berk L, Chang E, Chow E, Hahn C, Hoskin P, et al. Palliative radiotherapy for bone metastases: an ASTRO evidence-based guideline. Int J Radiat Oncol Biol Phys. 2011;79(4):965-76.

19. Costelloe CM, Chuang HH, Madewell JE, Ueno NT. Cancer response criteria and bone metastases: RECIST 1.1, MDA and PERCIST. J Cancer. 2010;1:80-92.

20. Lipton A, Costa L, Ali SM, Demers LM. Bone markers in the management of metastatic bone disease. Cancer Treat Rev. 2001;27(3):181-5.

21. Costa L, Demers LM, Gouveia-Oliveira A, Schaller J, Costa EB, de Moura MC, et al. Prospective evaluation of the peptide-bound collagen type I crosslinks $\mathrm{N}$-telopeptide and C-telopeptide in predicting bone metastases status. J Clin Oncol Off J Am Soc Clin Oncol. 2002 Feb 1;20(3):850-6.

22. Boyle WJ, Simonet WS, Lacey DL. Osteoclast differentiation and activation. Nature. 2003 May 15;423(6937):337-42.

23. Mundy GR. Metastasis to bone: causes, consequences and therapeutic opportunities. Nat Rev Cancer. 2002 Aug;2(8):584-93.

24. de la Piedra C, Alcaraz A, Bellmunt J, Meseguer C, Gómez-Caamano A, Ribal MJ, et al. Usefulness of bone turnover markers as predictors of mortality risk, disease progression and skeletal-related events appearance in patients with prostate cancer with bone metastases following treatment with zoledronic acid: TUGAMO study. Br J Cancer. 2013 Jun 25;108(12):2565-72.

25. Leeming DJ, Koizumi M, Byrjalsen I, Li B, Qvist P, Tankó LB. The relative use of eight collagenous and noncollagenous markers for diagnosis of skeletal metastases in breast, prostate, or lung cancer patients. Cancer Epidemiol Biomark Prev Publ Am Assoc Cancer Res Cosponsored Am Soc Prev Oncol. 2006;15(1):32-8

26. Lein M, Wirth M, Miller K, Eickenberg H-U, Weissbach L, Schmidt K, et al. Serial markers of bone turnover in men with metastatic prostate cancer treated with zoledronic acid for detection of bone metastases progression. Eur Urol. 2007:52(5):1381-7.

27. Choices in Methods for Economic Evaluation [Internet]. Haute Autorité de Santé. [cited 2020 Jul 18]. Available from: https://www.has-sante.fr/jcms/r_14 99251/en/choices-in-methods-for-economic-evaluation

28. Claxton K, Sculpher M, McCabe C, Briggs A, Akehurst R, Buxton M, et al. Probabilistic sensitivity analysis for NICE technology assessment: not an optional extra. Health Econ. 2005 Apr;14(4):339-47.

29. Mauskopf JA, Sullivan SD, Annemans L, Caro J, Mullins CD, Nuijten M, et al. Principles of good practice for budget impact analysis: report of the ISPOR task force on good research practices--budget impact analysis. Value Health J Int Soc Pharmacoeconomics Outcomes Res. 2007 Oct;10(5):336-47.

30. Palma DA, Olson R, Harrow S, Gaede S, Louie AV, Haasbeek C, et al. Stereotactic ablative radiotherapy for the comprehensive treatment of Oligometastatic cancers: long-term results of the SABR-COMET phase II randomized trial. J Clin Oncol Off J Am Soc Clin Oncol. 2020;2:JCO2000818.

31. Boevé LMS, Hulshof MCCM, Vis AN, Zwinderman AH, Twisk JWR, Witjes WPJ, et al. Effect on survival of androgen deprivation therapy alone compared to androgen deprivation therapy combined with concurrent radiation therapy to the prostate in patients with primary bone metastatic prostate Cancer in a prospective randomised clinical trial: data from the HORRAD trial. Eur Urol. 2019;75(3):410-8.

32. Clarke NW, Ali A, Ingleby FC, Hoyle A, Amos CL, Attard G, et al. Addition of docetaxel to hormonal therapy in low- and high-burden metastatic hormone sensitive prostate cancer: long-term survival results from the STAMPEDE trial. Ann Oncol Off J Eur Soc Med Oncol. 2019:30(12):19922003.

33. Ost P, Reynders D, Decaestecker K, Fonteyne V, Lumen N, De Bruycker A, et al. Surveillance or Metastasis-Directed Therapy for Oligometastatic Prostate Cancer Recurrence. A Prospective, Randomized, Multicenter Phase II Trial. J Clin Oncol Off J Am Soc Clin Oncol. 2018;36(5):446-53.

34. Radwan N, Phillips R, Ross A, Rowe SP, Gorin MA, Antonarakis ES, et al. A phase II randomized trial of observation versus stereotactic ablative 
Radiatlon for OLigometastatic prostate CancEr (ORIOLE). BMC Cancer. 2017; 17(1):453.

35. David S, Tan J, Savas P, Bressel M, Kelly D, Foroudi F, et al. Stereotactic ablative body radiotherapy (SABR) for bone only oligometastatic breast cancer: a prospective clinical trial. Breast Edinb Scotl. 2020;49:55-62.

36. Conibear J, Chia B, Ngai Y, Bates AT, Counsell N, Patel R, et al. Study protocol for the SARON trial: a multicentre, randomised controlled phase III trial comparing the addition of stereotactic ablative radiotherapy and radical radiotherapy with standard chemotherapy alone for oligometastatic non-small cell lung cancer. BMJ Open. 2018;8(4):e020690.

37. Al-Shafa F, Arifin AJ, Rodrigues GB, Palma DA, Louie AV. A review of Ongoing trials of stereotactic ablative radiotherapy for Oligometastatic cancers: where will the evidence Lead? Front Oncol. 2019;9:543.

\section{Publisher's Note}

Springer Nature remains neutral with regard to jurisdictional claims in published maps and institutional affiliations.

Ready to submit your research? Choose BMC and benefit from:

- fast, convenient online submission

- thorough peer review by experienced researchers in your field

- rapid publication on acceptance

- support for research data, including large and complex data types

- gold Open Access which fosters wider collaboration and increased citations

- maximum visibility for your research: over $100 \mathrm{M}$ website views per year

At $\mathrm{BMC}$, research is always in progress.

Learn more biomedcentral.com/submissions 